Pacific Journal of Mathematic 


\title{
A GOLDIE THEOREM FOR DIFFERENTIABLY PRIME RINGS
}

\author{
JOHN R. FISHER
}

The main goal of this paper is to prove analogues of the Goldie theorems for associative rings with derivations. It is shown that a differentiably prime ring, with suitable chain conditions, has a differentiably simple Artinian total ring of quotients, and, conversely, that a differential subring which is an order in a differentiably simple Artinian ring is a differentiably prime ring which has the chain conditions referred to above. A similar theorem concerning differentiably semiprime rings which are orders in differentiably semi-simple rings is also given.

Suppose that $A$ is an associative ring and that $D$ is a set of derivations of $A$, a derivation of $A$ being any function $d$ on $A$ such that $d(a+b)=d a+d b$, and $d(a b)=(d a) b+a(d b)$ for all $a, b \in A$. An ideal $I$ of $A$ is a D-ideal provided $d(I) \subseteq I$ for all $d \in D$. A is D-prime provided $H I=0$ implies $H=0$ or $I=0$ for any $D$-ideals $H$ and $I$ of $A$. $A$ is $D$-semiprime provided $A$ has no nonzero nilpotent $D$ ideals. $A$ is $D$-semisimple provided there are no nonzero $D$-ideals contained in the Jacobson radical of $A . \quad A$ is $D$-simple provided $A^{2} \neq 0$ and $A$ has no proper $D$-ideals. Finally, $A$ is said to be differentiably prime (resp. semiprime, semisimple, simple) provided $A$ is $D$-prime (resp. semiprime, semisimple, simple) for some set of derivations $D$, hence for the set $D=\operatorname{der} A$ of all derivations of $A$.

The ring $A$ is $D$-prime if and only if the left (right) annihilator of a nonzero left (right) $D$-ideal is zero. Another equivalent is that if $(\delta a) b\left(\delta^{\prime} c\right)=0$ for all products $\delta, \delta^{\prime}$ of derivations in $D$, and for every $b \in A$, then $a=0$ or $c=0$. Other easy consequences of the definitions are that differentiably prime implies differentiably semiprime, and that differentiably simple implies differentiably prime.

It is straightforward to show, using arguments suitable for prime rings, that every differentiably prime ring has characteristic zero or a prime number. If $A$ is a differentiably prime ring of characteristic zero, and satisfies a suitable chain condition (Corollary to Lemma 2 in $\$ 2$ below), then $A$ must be a prime ring. This is analogous to the fact that a differentiably simple ring of characteristic zero with a minimal ideal must be simple [3, Corollary to Thm. 4].

The ring $Q$ is a total ring of left quotients for the ring $A$ provided $A \subseteq Q$, every nonzero-divisor (regular element) in $A$ is invertible in $Q$, 
and each $q \in Q$ can be written $q=b^{-1} a$ for some $a, b \in A$, with $b$ a regular element in $A$. It will also be said that $A$ is a subring and left order in $Q$. $A$ has a total ring of left quotients if and only if $A$ satisfies the (left) Öre condition: $a, b \in A$ with $b$ regular implies there exist $c, e \in A$ with $e$ regular such that $e a=c b$.

The following fact is crucial to the main theorems of this paper: Suppose that $A$ has a total ring of left quotients $Q$, and that $d$ is any derivation of $A$. Then $d$ extends in a unique fashion to give a derivation $\hat{d}$ of $Q$. If $q \in Q$ is written $q=b^{-1} a$, then $\hat{d} q=$ $-b^{-1}(d b) b^{-1} a+b^{-1}(d a)$. To prove this, it must be shown that $\hat{d}$ is well-defined, additive, and obeys the product rule on $Q$. This is tedious, but can be done using the Öre condition and the definition of $\hat{d}$. Note that $\hat{d} b^{-1}=-b^{-1}(d b) b^{-1}$, which will be found to be necessary if $\hat{d}$ is applied to both sides of the equation $b^{-1} b=1$, and one solves for $\hat{d} b^{-1}$. If $A$ has a total ring of left quotients $Q$ and $D$ is a set of derivations of $A$, let $\hat{D}=\{\hat{d} \mid d \in D\}$ where $\hat{d}$ is the extension to $Q$ as above.

$A$ is a left Goldie ring provided $A$ has no infinite ascending chain of left annihilators, and no infinite direct sum of left ideals. The two main theorems of this paper can now be stated. The proofs are in $\$ 2$.

THEOREM 1. Assume that $A$ is a D-prime ring, that the nil radical $N$ of $A$ is nilpotent, that $A$ satisfies the ascending chain condition on right annihilators, and that both $A$ and $A / N$ are left Goldie rings. Then $A$ has a total ring of left quotients $Q$ which is a $\hat{D}$-simple, left Artinian ring. Conversely, assume that $A$ is a $\hat{D}$-closed subring and left order in the $\hat{D}$-simple, left Artinian ring $Q$. Then $A$ is $D$-prime, where $D=$ $\left\{\left.\hat{d}\right|_{A} \mid \hat{d} \in \hat{D}\right\}$, the nil radical $N$ of $A$ is nilpotent, $A$ satisfies the ascending chain condition on right annihilators, and both $A$ and $A / N$ are left Goldie rings.

Theorem 2. Assume that $A$ is a D-semiprime, left Noetherian ring. Then $A$ has a total ring of left quotients $Q$ which is $a \hat{D}$ semisimple, left Artinian ring. As a partial converse, if $A$ is a $\hat{D}$-closed subring and left order in the $\hat{D}$-semisimple, left Artinian ring $Q$, then $A$ is D-semiprime, where $D=\left\{\left.\hat{d}\right|_{A} \mid \hat{d} \in \hat{D}\right\}$.

If $A$ has characteristic zero then the statements of Theorems 1 and 2 reduce to versions of the Goldie theorems. This observation uses the Corollary to Lemma 2 proved in $\S 2$. Of course, if $D$ is the empty set, then the theorems again reduce to the Goldie theorems. It should be noted, however, that the Goldie theorems (and proofs contributed by various authors) are used in the proofs of Theorems 1 and 2 . 
A differentiably simple ring $Q$ with a minimal ideal is either simple or there exists a simple ring $S$ of prime characteristic, and a positive integer $n$, such that $Q \cong S \otimes B_{n}$ where the tensor product is over $Z_{p}$ ( $p$ the characteristic of $S$ ) and $B_{n}$ denotes the (commutative associative with unit) truncated polynomial algebra $Z_{p}\left[X_{1}, \cdots, X_{n}\right] /\left(X_{1}{ }^{P}, \cdots, X_{n}{ }^{P}\right)$, by [1, Main Theorem]. Hence $Q$ is Artinian if and only if $S$ is. An example of a differentiably prime, but not prime, ring would be one of the form $P \otimes B_{n}$ where $P$ is a subring and left order in $S$.

A differentiably semisimple, left Artinian ring $Q$ is a direct sum $Q=Q_{1} \oplus \cdots \oplus Q_{k}$ where the $Q_{i}$ are differential ideals of $Q$ and each $Q_{i}$ is a differentiably simple, left Artinian ring [1, Th. 8.2, Cor. 8.3]. Using this expression for $Q$ and the type of example used for a differentiably prime ring in the previous paragraph, one can construct examples of differentiably semiprime rings which are not differentiably prime.

The left orders in a simple Artinian ring are characterized in the Faith-Utumi Theorem. In analogy, it could be asked whether a differential subring and left order in $S \otimes B_{n}$ need be of the form $P \otimes B_{n}$ where $P$ is a subring and left order in $S$. The following is an example, for any prime characteristic $p$, of a commutative differential subring and order $A$ in a differentiably simple Artinian ring of the form $B_{1}(E)$, where $E$ is some field of characteristic $p$, such that $A$ is not of the form $B_{1}(I)$ for any subring $I$ of $A$ which is an integral domain consisting of differential constants of $A$.

Let $B=Z_{p}[u, v]$, the polynomial ring in commuting indeterminants $u$ and $v$, and let $A=B[(u / v)+x]$ considered as a subring of $B_{1}(E)$ where $E$ is the field of quotients of $B$. Consider $B_{1}(E)$ as $E[x]$ where $x^{p}=0$ and let $d=d / d x$ denote differentiation by $x$. Then $A$ is a $d$-subring and order in $B_{1}(E)$, but straightforward calculation shows that $A$ cannot be written $B_{1}(I)$ for any suitable $I$ described at the end of the discussion in the preceding paragraph.

\section{Proofs of the theorems.}

Lemma 1. Assume that $A$ is a D-semiprime ring and that the nil radical $N$ of $A$ is nilpotent. Then $a+N$ is regular in $A / N$ implies that a is regular in $A$.

Lemma 2. Assume that $A$ is a $D$-prime ring, that the nil radical $N$ of $A$ is nilpotent, and that $A$ has the ascending chain condition on left annihilators and on right annihilators. Then the ideal divisors of zero of $A$ are nilpotent, $A / N$ is a prime ring, and a regular in $A$ implies that $a+N$ is regular in $A / N$. 
Corollary. If, in addition to the hypotheses in Lemma 2, A is assumed to have characteristic zero, then A must be a prime ring.

Proof of Lemma 1. Suppose that $a+N$ is regular in $A / N$ but that $a b=0$ for some $b$ in $A$. Then $b \in N$, and, for any derivation $d$ of $A$, $0=d(a b)=(d a) b+a(d b)$, or $a(d b)=-(d a) b \in N$, and so $d b \in N$, since again $a+N$ is regular in $A / N$. This proves the first step of the following induction. Suppose $\delta b \in N$ for every string of derivations $\delta=d_{1} \cdots d_{k}$ of length $k$. If a string of derivations of length $k+1$ is written in the form $d \delta$ where $d$ is a derivation and $\delta$ is a string of length $k$, then $0=d \delta(a b)=\Sigma\left(\delta_{1} a\right)\left(\delta_{2} b\right)+a(d \delta b)$ where $\delta_{1}, \delta_{2}$ are strings of derivations of length at most $k$, hence each $\delta_{2} b \in N$, and so $a(d \delta b) \in N$ and so $d \delta b \in N$. Thus, if $\delta$ is any string of derivations of $A$, then $\delta b \in N$. This implies that the differential ideal $I$ of $A$ generated by $b$ is contained in $N$, hence that $I$ is a nilpotent differential ideal of $A$. Since $A$ is $D$-semiprime for some set of derivations $D$, it must be the case that $I=0$, hence $b=0$. A similar argument shows that $b a=0$ implies that $b=0$.

Proof of Lemma 2. Suppose that $a B=0$, where $a$ is a nonzero element of $A$, and $B$ is an ideal of $A$. Then $B \supseteq B^{2} \supseteq B^{3} \supseteq \cdots$ is a descending chain of ideals of $A$ and $\ell(B) \subseteq \ell\left(B^{2}\right) \subseteq \ell\left(B^{3}\right) \subseteq \cdots$ where $\ell(B)$ denotes the left annihilator of $B$ in $A$. If $U$ is the union of all the $\ell\left(B^{i}\right)$, then $U$ is a differential ideal of $A$, since if $x \in \ell\left(B^{i}\right)$ then $d x \in \ell\left(B^{i+1}\right)$ for any derivation $d$ of $A$. Since $A$ has the ascending chain condition on left annihilators, $U=\ell\left(B^{k}\right)$ for some $k$, and since $a \in \ell\left(B^{k}\right), \ell\left(B^{k}\right) \neq 0$. But $\ell\left(B^{k}\right) B^{k}=0$, and since $A$ is $D$-prime for some set of derivations $D$, it must be the case that $B^{k}=0$. A similar argument using the ascending chain condition on right annihilators shows that $B a=0$ implies $B$ is nilpotent.

The proof that $A / N$ is a prime ring is straightforward.

Now suppose that $a$ is regular in $A$ but that $a b \in N$ for some $b$ in $A$. If $N=0$, then $a b=0$, so $b=0$. If $N \neq 0$, then $a b N^{k-1}=0$ where $N^{k-1} \neq 0, N^{k}=0$. Thus $b N^{k-1}=0$ since $a$ is regular, so $b$ is contained in an ideal divisor of zero, hence $b \in N$. Similarly, $b a \in N$ implies $b \in N$.

Proof of Corollary. By [3, Proof of Thm. 4], if $A$ is a primary ring (ideal divisors of zero are nilpotent) whose additive group is torsion free, then $d a$ lies in a nilpotent ideal if $a$ does, for every derivation $d$ of $A$. This would imply that $N$ is a nilpotent differential ideal, hence $N=0$, since $A$ is $D$-prime for some set of derivations $D$. Thus $A \cong A / N$ is prime by Lemma 2 . 
Proof of Theorem 1. Assume the hypotheses on $A$ in the statement of the theorem. The proof of the first part of the theorem is separated into parts.

(i) The existence of $Q$. For this it is shown that $A$ satisfies the Öre condition. By Lemmas 1 and 2, it follows that $\bar{a}=a+N$ is regular in $\bar{A}=A / N$ if and only if $a$ is regular in $A$, and that $\bar{A}$ is a prime Goldie ring. First we show that if $a, b \in A$ are both regular then there exist $c, e \in A$ with $c$ regular such that $e a=c b$. Let $M=$ $\{x \in A \mid x b \in A a\}$. We claim that $\bar{M}$, the image of $M$ in $\bar{A}$, is an essential left ideal of $\bar{A}$. Note that $\bar{M}$ is essential in $\bar{A}$ if and only if for every left ideal $L$ of $A, M \cap L \subseteq N$ implies $L \subseteq N$. So suppose that $M \cap L \subseteq N$. Then $A a \cap L b \subseteq N$ since if $y \in A a \cap L b$, then $y=u a=$ $v b$ or $v \in M \cap L \subseteq N$, thus $y=v b \in N$. But $\overline{A a}=\bar{A} \bar{a}$ is essential in $\bar{A}$ by $[2$, Lemma 7.2 .3$, p. 174], since $\bar{a}$ is regular in $\bar{A}$, and $\bar{A}$ is a prime Goldie ring. Hence $L b \subseteq N$. Since $b$ is regular in $A, L \subseteq$ $N$. Therefore $\bar{M}$ is an essential left ideal in $\bar{A}$, hence there exists a $\bar{c} \in \bar{M}$ with $\bar{c}$ regular in $\bar{A}$ by [2, Lemma 7.2 .5 , p. 175]. Thus there exists a $c$ regular in $A$ such that $c b=e a$ for some $e \in A$.

Next, the above is used to show that the full Öre condition holds for $A$. Suppose $a, b \in A$ with $b$ regular in $A$. Since $\bar{A}$ is a prime Goldie ring, and hence satisfies the Öre condition, there exist $\bar{e}, \bar{c}$ in $\bar{A}$ with $\bar{e}$ regular in $\bar{A}$, so $e$ is regular in $A$, such that $\bar{e} \bar{a}=\bar{c} \bar{b}$ or $e a=c b+n$ for some $n \in N$. Let $u=c-e$, and write $e a=u b+(e b+n)$. Now $(e b+n)+N=e b+N$ is regular in $\bar{A}$ so $e b+n$ is regular in $A$. Using the preceding paragraph, there are $r, s$ in $A$ with $r$ regular in $A$ such that $r(e b+n)=s b$. Hence $r e a=r u b+r(e b+n)=r u b+s b=(r u+s) b$ or $(r e) a=(r u+s) b$ which gives the Öre condition since $r e$ is regular in A.

(ii) If $N_{1}$ is the nil radical of $Q$, then $N_{1}=Q N$ and $N=$ $N_{1} \cap A . \quad N_{1} \cap A$ is a nil ideal of $A$ so $N_{1} \cap A \subseteq N$. Since $(Q N)^{k} \subseteq$ $Q N^{k}$ and $N$ is nilpotent, $Q N$ is nilpotent, thus $Q N \subseteq N_{1}$, and so $N \subseteq Q N \subseteq N_{1}$. Hence $N=N_{1} \cap A$. Now $N_{1} \subseteq Q N$ since if $b^{-1} m \in N_{1}$, then $b\left(b^{-1} m\right)=m \in N_{1} \cap A=N$, and so $b^{-1} m \in Q N$. Hence $N_{1}=Q N$.

(iii) $A / N$ is a prime Goldie ring whose total ring of left quotients is isomorphic to $Q / N_{1}$. Hence $Q / N_{1}$ is a simple, left Artinian ring. Let $Q(A / N)$ denote the total ring of left quotients of $A / N$, and let $\phi$ be the map from $Q$ to $Q(A / N)$ defined by $\phi\left(b^{-1} a\right)=(b+N)^{-1}(a+N)$. It is straightforward to show that $\phi$ is well-defined and a homomorphism onto $Q(A / N)$ with kernel $N_{1}$.

(iv) $Q$ is $\hat{D}$-simple. Suppose that $I$ is a nonzero $\hat{D}$-ideal of $Q$. Then $I \cap A$ is a nonzero $D$-ideal of $A$. $I \cap A \not \subset N$ since $A$ is 
$D$-prime. Hence $I \notin \subset N_{1}$. Thus $\left(I+N_{1}\right) / N_{1}$ is a nonzero ideal of $Q / N_{1}$. Since $Q / N_{1}$ is simple, $I+N_{1}=Q$. If $I \neq Q$ then $I$ contains only non-invertible elements of $Q$. From the statement $Q=I+N_{1}$ it will be shown that no element of $Q$ is invertible, which is not possible, hence $I=Q$. Now suppose that $x \in I+N_{1}$ is invertible in $Q$, $I \neq Q$. Write $x=a^{-1} b+n$ where $n \in N_{1}$ and $a^{-1} b \in I$. Since $a^{-1} b$ is not invertible, $b$ is not regular in $A$. So there exists a nonzero $z$ in $A$ such that $b z=0$ or $z b=0$. The element $a x=b+a n$ must also be invertible. Using $b z=0, a x z=a n z \in N_{1}$. Since $a x$ is invertible, $z \in N_{1}$. Let $z^{k}$ be the smallest nonzero power of $z$ such that $a n z^{k}=$ 0 . Then $a x z^{k}=b z^{k}+a n z^{k}=0$, which is impossible since $a x$ is invertible and $z^{k} \neq 0$. A similar argument can be given for the case $z b=$ 0 . Hence no element of $I+N_{1}$ can be invertible if $I \neq Q$.

(v) $Q$ is left Artinian. This will be proved by showing that $Q$ has a minimal ideal. For in that case $Q$ is a differentiably simple ring with a minimal ideal. Thus by [1, Main Theorem], $Q$ must either be simple, or there exists a simple ring $S$ of prime characteristic and a positive integer $n$ such that $Q \cong S \otimes B_{n}$. If $Q$ is simple, then $N=N_{1}=0$, so $Q \cong$ $Q / 0 \cong Q(A / 0)$ is left Artinian since in this case $A$ is a prime ring. On the other hand, if $Q=S \otimes B_{n}$, then $S \cong Q / N_{1}$, so $S$ is Artinian by (iii), and so $Q=S \otimes B_{n}$ is also left Artinian.

It can be assumed that $Q$ is not simple. Hence $H=$ $\left\{q \in Q \mid N_{1} q=0\right\}$ contains a nonzero element $m$. Then $Q m Q$ is a nonzero left $Q / N_{1}$-module using the action $\bar{q} y=q y$ for all $q \in Q, y \in Q m Q$. This action is well-defined since $N_{1}(Q m Q)=$ 0 . Moreover, the $Q / N_{1}$-submodules of $Q m Q$ are just those left ideals of $Q$ contained in $Q m Q$. $Q / N_{1}$ is a simple, left Artinian ring, so $Q m Q$ is a completely reducible left $Q / N_{1}$-module. Hence $Q m Q$ is a direct sum of minimal left ideals of $Q$. But $Q$ contains no infinite direct sum of left ideals since $A$ does not. Hence $Q m Q$ is a finite direct sum of minimal left ideals of $Q$, so $Q m Q$ is an Artinian left $Q / N_{1^{-}}$ module. Therefore, $Q m Q$, being an ideal of $Q$ itself, must contain a minimal ideal of $Q$.

Now assume the hypotheses on $Q$ and $A$ stated in the converse of the theorem. Then $A$ is $D$-prime by an argument similar to one that can be given to show that a subring and left order in a simple Artinian ring must be prime, as in [2, Thm. 7.2.3, p. 177]. That the nil radical $N$ of $A$ is nilpotent, and that both $A$ and $A / N$ are Goldie rings follows from [4, Part II, §2]. Since $Q$ is left Artinian, $Q$ satisfies the descending chain condition on left annihilators, hence so does $A$ since $A$ is a left order in $Q$. Thus $A$ satisfies the ascending chain condition on right annihilators. This shows that $\boldsymbol{A}$ must satisfy all of the chain conditions used in the first part of the theorem. 
Proof of Theorem 2. If $A$ is a $D$-semiprime, left Noetherian ring then, using Lemma 1 and [4, Part II, \$1], $A$ has a left Artinian total ring of left quotients $Q$.

To show that $Q$ is $\hat{D}$-semisimple, suppose that $H$ is a $\hat{D}$-ideal of $Q$ contained in the Jacobson radical of $Q$. Since $Q$ is left Artinian, the Jacobson radical of $Q$ is nilpotent. Thus $H$ is a nilpotent $\hat{D}$-ideal of $Q$. Hence $H \cap A$ is a nilpotent $D$-ideal of $A$, and so $H \cap A=$ 0 . Consequently, $H=0$, and thus $Q$ is $\hat{D}$-semisimple.

For the partial converse, assume that $A$ is a $\hat{D}$-closed subring and left order in the $\hat{D}$-semisimple, left Artinian ring $Q$, and let $D=$ $\left\{\left.d\right|_{A} \mid d \in \hat{D}\right\}$. Then $Q=Q_{1} \oplus \cdots \oplus Q_{k}$ where the $Q_{i}$ are $\hat{D}$-ideals of $Q$ and $\hat{D}$-simple rings, by [1, Thm. 8.2, Cor. 8.3], so that any $\hat{D}$-ideal of $Q$ must be a sum of some of the $Q_{i}$. To show now that $A$ must be $D$-semiprime, use an argument just like one that can be given to show that a subring and left order in a semisimple, left Artinian ring must be semiprime [2, Thm. 7.2, p. 177], only using differential ideals instead of ordinary ideals.

\section{REFERENCES}

1. R. E. Block, Determination of the differentiably simple rings with a minimal ideal, Annals of Math., 90 (1969), 433-459.

2. I. N. Herstein, Noncommutative Rings, MAA Carus Monographs, \# 15, 1968.

3. E. C. Posner, Differentiably simple rings, Proc. Amer. Math. Soc., 11 (1960), 337-343.

4. L. W. Small, Orders in Artinian rings, J. Algebra, 4 (1966), 13-41.

Received December 3, 1973 and in revised form July 5, 1974.

California State Polytechnic University, Pomona 



\section{CONTENTS}

Zvi Artstein and John A. Burns, Integration of compact set-valued functions

J. A. Beachy and W. D. Blair, Rings whose faithful left ideals are cofaithful

Mark Benard, Characters and Schur indices of the unitary reflection group $[321]^{3}$

H. L. Bentley and B. J. Taylor, Wallman rings ............................. 15

E. Berman, Matrix rings over polynomial identity rings II ............... 37

Simeon M. Berman, A new characterization of characteristic functions of absolutely continuous distributions ........................ 323

Monte B. Boisen, Jr. and Philip B. Sheldon, Pre-Prüfer rings ............ 331

A. K. Boyle and K. R. Goodearl, Rings over which certain modules are injective

J. L. Brenner, R. M. Crabwell and J. Riddell, Covering theorems for finite nonabelian simple groups. $V$

H. H. Brungs, Three questions on duo rings .................................... 345

Iracema M. Bund, Birnbaum-Orlicz spaces of functions on groups ....351

John D. Elwin and Donald R. Short, Branched immersions between 2-manifolds of higher topological type

J. K. Finch, The single valued extension property on a Banach space ................................................................................. 61

J. R. Fisher, A Goldie theorem for differentiably prime rings ............ 71

Eric M. Friedlander, Extension functions for rank 2, torsion free abelian groups ...................................................................... 371

J. Froemke and R. Quackenbusch, The spectrum of an equational class of groupoids

B. J. Gardner, Radicals of supplementary semilattice sums of

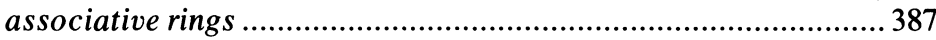

Shmuel Glasner, Relatively invariant measures ...............................393

G. R. Gordh, Jr. and Sibe Mardešić, Characterizing local connectedness in inverse limits...

S. Graf, On the existence of strong liftings in second countable topological spaces

S. Gudder and D. Strawther, Orthogonally additive and orthogonally increasing functions on vector spaces ........................................427

F. Hansen, On one-sided prime ideals .......................................... 79

D. J. Hartfiel and C. J. Maxson, A characterization of the maximal monoids and maximal groups in $\beta x$.

Robert E. Hartwig and S. Brent Morris, The universal flip matrix and the generalized faro-shuffle 


\section{Pacific Journal of Mathematics}

Vol. 58, No. 1

March, 1975

John Allen Beachy and William David Blair, Rings whose faithful left ideals are cofaithful .................................... 1

Herschel Lamar Bentley and Barbara June Taylor, Wallman rings ........ 15

Elizabeth Berman, Matrix rings over polynomial identity rings. II ...... 37

Ann K. Boyle and Kenneth R. Goodearl, Rings over which certain modules are injective ................................. 43

J. L. Brenner, Robert Myrl Cranwell and James Riddell, Covering theorems for finite nonabelian simple groups. $V \ldots \ldots \ldots \ldots \ldots \ldots \ldots \ldots \ldots$

James Kenneth Finch, The single valued extension property on a Banach

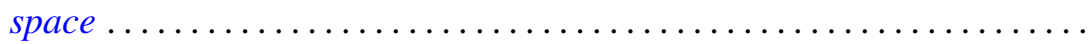

John Robert Fisher, A Goldie theorem for differentiably prime rings........ Friedhelm Hansen, On one-sided prime ideals .................... Jon Craig Helton, Product integrals and the solution of integral equations..........................................

Barry E. Johnson and James Patrick Williams, The range of a normal

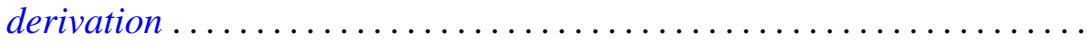

Kurt Kreith, A dynamical criterion for conjugate points ............ 123

Robert Allen McCoy, Baire spaces and hyperspaces .............. 133

John McDonald, Isometries of the disk algebra ................ 143

H. Minc, Doubly stochastic matrices with minimal permanents ......... 155

Shahbaz Noorvash, Covering the vertices of a graph by vertex-disjoint paths. ...

Theodore Windle Palmer, Jordan *-homomorphisms between reduced Banach*-algebras

Donald Steven Passman, On the semisimplicity of group rings of some

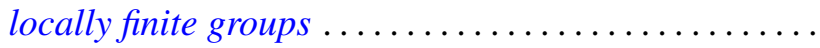

Mario Petrich, Varieties of orthodox bands of groups .

Robert Horace Redfield, The generalized interval topology on distributive

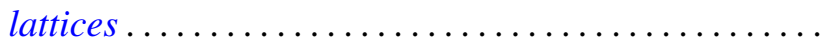

James Wilson Stepp, Algebraic maximal semilattices .... . .

Patrick Noble Stewart, A sheaf theoretic representation of rings with Boolean orthogonalities ........................

Ting-On To and Kai Wing Yip, A generalized Jensen's inequality......... 255

Arnold Lewis Villone, Second order differential operators with self-adjoint

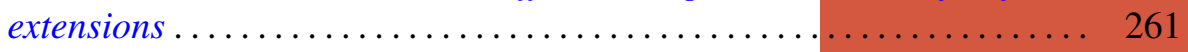

Martin E. Walter, On the structure of the Fourier-Stieltjes algebra ....... 267

John Wermer, Subharmonicity and hulls .................... 283

Edythe Parker Woodruff, A map of $E^{3}$ onto $E^{3}$ taking no disk onto a

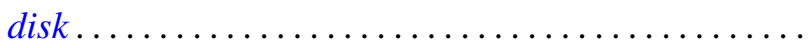

